\title{
Novel BRCA1 deleterious mutation (c.1949_1950delTA) in a woman of Senegalese descent with triple- negative early-onset breast cancer
}

\author{
ORLAND DIEZ ${ }^{1,2}$, AMADEU PELEGRÍ ${ }^{3 *}$, NEUS GADEA ${ }^{4}$, SARA GUTIÉRREZ-ENRÍQUEZ ${ }^{2}$, \\ MIRIAM MASAS $^{2}$, ANNA TENÉS ${ }^{2}$, NINA BOSCH ${ }^{4}$, JUDITH BALMAÑA ${ }^{4}$ and BEGOÑA GRAÑA ${ }^{4}$ \\ ${ }^{1}$ Oncogenetics Laboratory, University Hospital Vall d'Hebron; ${ }^{2}$ Vall d'Hebron Institute of Oncology, Barcelona; \\ ${ }^{3}$ Medical Oncology Service, Hospital Sant Pau i Santa Tecla, Tarragona; \\ ${ }^{4}$ Medical Oncology Department, University Hospital Vall d'Hebron, Barcelona, Spain
}

Received April 1,2011; Accepted August 11, 2011

DOI: $10.3892 / \mathrm{ol} .2011 .390$

\begin{abstract}
Limited information exists regarding $B R C A l$ and $B R C A 2$ genetic testing and genetic diversity in $B R C A 1$ and $B R C A 2$ in sub-Saharan African populations. We report a novel mutation that consists of a deletion of 2 bp (c.1949_1950delTA) in the exon 11 of the BRCA1 gene. This is a frameshift mutation that causes the disruption of the translational reading frame resulting in a premature stop codon downstream in the BRCA1 protein. The mutation was present in a Senegalese woman with a triple-negative breast tumor and a family history of breast cancer.
\end{abstract}

\section{Introduction}

Breast cancer is the most commonly occurring cancer among women. A family history of breast cancer is one of the main risk factors for developing the disease. It is currently estimated that approximately $5-10 \%$ of breast cancers are due to an inherited predisposition, and approximately $20-25 \%$ of this risk is explained by two high-penetrance susceptibility genes: BRCA1 (17q21) (MIM no. 113705) (1) and BRCA2 (13q12-13) (MIM no. 600185) (2). Both are tumor suppressor genes involved mainly in DNA repair. Mutations in $B R C A 1$ or $B R C A 2$ account for a lifetime risk of approximately $40-65 \%$ for breast cancer and $11-40 \%$ for ovarian cancer (3). Extensive analyses of the $B R C A 1$ and $B R C A 2$ genes have resulted in the identification of a large number of different disease-causing germline mutations (4).

Correspondence to: Dr Orland Diez, Oncogenetics Laboratory, University Hospital Vall d'Hebron, Vall d'Hebron Institute of Oncology, Barcelona, Spain

E-mail: Odiez@vhebron.net

\section{*Deceased}

Key words: African, BRCA1, familial cancer, genetic predisposition, hereditary breast cancer, mutation
However, limited information exists regarding $B R C A l$ and $B R C A 2$ genetic testing in the majority of non-Caucasian ethnicities and there is a paucity of data regarding the genetic diversity in BRCA1 and BRCA2 in populations of African descent. We report a novel mutation that consists of a deletion of 2 bp (c.1949_1950delTA) in the exon 11 of the BRCAI gene. This is a frameshift mutation that causes the disruption of the translational reading frame, resulting in a stop codon downstream in the 671 position of the BRCA 1 protein. The mutation was present in a Senegalese woman with triplenegative breast cancer and a family history of the disease.

\section{Patient and methods}

Patient. As part of genetic studies carried out on breast/ovarian families in the University Hospital Vall d'Hebron (Barcelona, Spain), germ-line BRCAl and BRCA2 mutations were screened in the proband of the family reported in this study.

The proband was a 33 year-old premenopausal Senegalese female of Wolof ethnicity, diagnosed with a triple-negative (ER-, PgR-, HER2-) stage IV breast ductal carcinoma. She had six male and four female siblings. One sister, a maternal cousin and a maternal aunt succumbed to breast cancer at the ages of 47, 50, and approximately 70, respectively (Fig. 1 shows the pedigree of the affected family). The proband underwent genetic counseling and signed informed consent for gene testing of germ-line BRCA1 and BRCA2 mutations. The study was approved by the ethics committee of the University Hospital Vall d'Hebron.

Methods. Genomic DNA was extracted from whole blood using a Puregene Genome DNA purification kit (Gentra System, Minneapolis, MN, USA). DNA was amplified by PCR using primers specific for the coding sequence and intron/exon boundaries of the two genes. Analysis of the entire coding and flanking sequences was carried out by sequencing using a BigDye terminator V3.1 cycle sequencing kit on a 3130xl Genetic Analyzer (Applied Biosystems, Foster City, CA, USA). Both forward and reverse strands were sequenced. The variant sequences described in our study were named according to GenBank (BRCA1 NM_007294; BRCA2 NM_000059). 


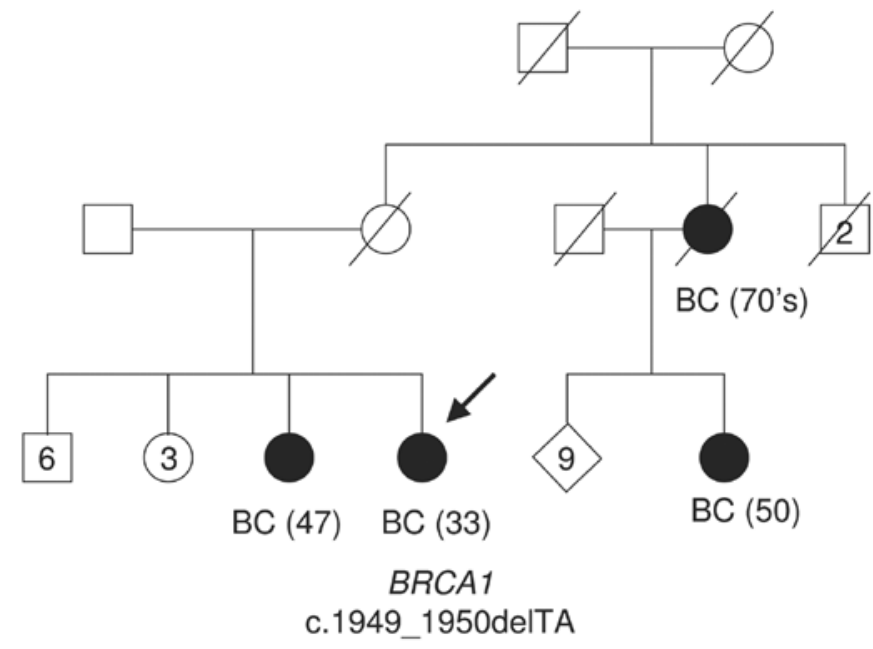

Figure 1. Family pedigree. Numbers in parentheses indicate the age at the time of diagnosis; arrow indicates proband. BC, breast cancer.

\section{Results}

We identified in the exon 11 of the BRCAl gene the novel mutation c.1949_1950delTA (p.Ile650LysfsX22) (HGVS nomenclature) or 2068delTA (BIC database nomenclature) (Fig. 2) in the index case of a family with hereditary breast cancer from Senegal.

To the best of our knowledge, this two-base pair deletion is not present in the BIC database and has not previously been published. Furthermore, the index case presented two unclassified variants in BRCA2. The first is the novel variant c.296-7dupT in the intron 2 of the gene, which is localized in the poly $\mathrm{T}(10)$ of the splicing acceptor site. This variant may cause the same effect as the IVS2-7T >A known variant, which generates an alternative transcript with the in-frame skipping of the exon 3 , and is not considered to be completely pathogenic due to its presence in healthy populations (5). An RNA sample from the proband and DNA from other family members to study this variant were not available. The second variant, c.5710C $>\mathrm{G}$ in exon 11 (5938C $>\mathrm{G}$, BIC nomenclature), causes the p.L1904V change in the BRCA2 protein. This variant has been reported eight times in the BIC database. Although it has a weak probability of altering splicing or protein functionality (Alamut Software 1.54, Interactive Biosoftware), this variant remains unclassified.

\section{Discussion}

The majority of studies on BRCA1 and BRCA2 genes have been performed in Caucasian women of European descent, while little information exists regarding other ethnicities. Nevertheless, several reports have described BRCAl and $B R C A 2$ sequence variants in women of African or AfricanAmerican descent and have characterized a different spectrum of BRCA1 and BRCA2 mutations from that of the North American or European Caucasian population $(1,6-18)$.

Such studies have revealed ancient mutations originating in the African continent, such as the BRCAl recurrent 943ins10 mutation. Haplotype analyses of 943 ins 10

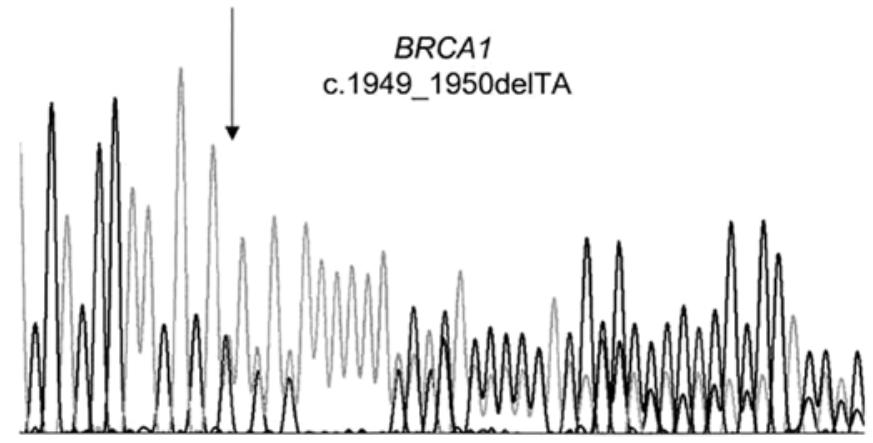

Figure 2. Electropherogram of the forward sequence of the region of the BRCA1 exon 11, which includes the wild-type and the mutated c.1949_1950delTA allele.

(c.815_824dup10) carriers indicate that this mutation, which has been identified in African-American and Latin-American/ Caribbean families, has an ancient African origin $(8,13)$ in the Ivory Coast in West Africa (7). However, it is unclear whether some of the mutations reported are unique to the African-American population. Of note, the 3036del4 mutation (c.2808_2811delACAA) in BRCA2, which was recurrently present in different European populations (19) has also been identified in young African women with breast cancer from Ibadan, Nigeria (14).

As of the end of 2010, the BIC database contains 38 BRCAI and 31 BRCA2 different deleterious mutations and a high number of sequence variants of unknown significance identified in families of African descent. Moreover, other mutations and variants identified in African/African-American families have also been published. These data suggest a significant level of genetic variation in BRCA1 and BRCA2 in populations of African descent. The novel c.1949_1950delTA mutation reported in the present study extends the knowledge of genetic susceptibility to breast cancer in sub-Saharan African populations. Furthermore, the finding of this mutation in a Senegalese family can be of interest for other populations originating from western Africa, as is the case for the vast majority of African-Americans.

The incidence and the age of onset of breast cancer vary across geographic regions and ethnic groups. Incidence and average age of onset are lower in African-American women than among Caucasian women $(20,21)$. These differences may be attributed to multiple environmental factors. Breast cancer incidence has increased in the last decades in African populations (22), most likely due to the better reporting and the adoption of a Western lifestyle in urban cities. However, biological and genetic factors may also explain variability between ethnicities. Studies in the United States have documented that breast cancers in young pre-menopausal African-American women were more aggressive, leading to a decrease in the overall survival rates compared to Caucasian women (23-25).

However, little data is currently available concerning the prevalence of $B R C A 1 / 2$ mutations among breast cancer patients indigenous to sub-Saharan countries. With the exception of the Ashkenazi population, African-American women 
diagnosed with breast cancer under the age of 35 years have been reported to have a higher prevalence of $B R C A 1$ mutations than any age-matched racial/ethnic group (26). The higher BRCAl mutation prevalence correlates with their elevated rates of triple-negative or basal-like breast cancer profile (27). These phenotypic traits are present in the female Senegalese patient carrying the BRCA1 c.1949_1950delTA mutation described in our report, who was diagnosed at 33 years of age with an ER-, PgR-, Her2- breast tumor.

Bearing in mind that there is a significant racial/ethnic variation in the spectrum of $B R C A 1 / 2$ mutations, new data regarding $B R C A 1$ and $B R C A 2$ genetic mutation prevalence and penetrance in African women is likely to improve the performance of risk-assessment tools and mutation-prediction models, and affect the clinical genetic testing process and follow-up for individuals of African ancestry (28).

\section{Acknowledgements}

This article is dedicated to Dr A. Pelegrí, who passed away during the preparation of the manuscript.

\section{References}

1. Miki Y, Swensen J, Shattuck-Eidens D, et al: A strong candidate for the breast and ovarian cancer susceptibility gene BRCA1. Science 266: 66-71, 1994.

2. Wooster R, Bignell G, Lancaster J, et al: Identification of the breast cancer susceptibility gene BRCA2. Nature 378: 789-792, 1995.

3. Antoniou A, Pharoah PDP, Narod S, et al: Average risks of breast and ovarian cancer associated with BRCA1 or BRCA2 mutations detected in case series unselected for family history: a combined analysis of 22 studies. Am J Hum Genet 72: 1117-1130, 2003

4. BIC http://research.nhgri.nih.gov/bic

5. Santarosa M, Viel A and Boiocchi M: Splice variant lacking the transactivation domain of the BRCA2 gene and mutations in the splice acceptor site of intron. Genes Chromosomes Cancer 26: 381-382, 1999.

6. Futreal PA, Liu Q, Shattuck-Eidens D, et al: BRCA1 mutations in primary breast and ovarían carcinomas. Science 266: 120-122, 1994.

7. Arena JF, Smith S, Plewinska M. et al: BRCAl mutations in African American women (abstract). Am J Hum Genet [Suppl] 59: A34, 1996.

8. Stoppa-Lyonnet D, Laurent-Puig P, Essioux L, et al: BRCA1 sequence variations in 160 individuals referred to a breast/ ovarian family cancer clinic. Institut Curie Breast Cancer Group. Am J Hum Genet 60: 1021-1030, 1997.

9. Gao Q, Neuhausen S, Cummings S, et al: Recurrent germ-line BRCA1 mutations in extended African American families with early-onset breast cancer. Am J Hum Genet 60:1233-1236, 1997.
10. Newman B, Mu H, Butler LM, et al: Frequency of breast cancer attributable to BRCA1 in a population-based series of American women. JAMA 279: 915-921, 1998.

11. Panguluri RCK, Brody LC, Modali R, et al: BRCA1 mutations in African Americans. Hum Genet 105: 28-31, 1999.

12. Dangel J, Wagner-Costalas J, Bove B, et al: Novel germline BRCA1 mutation (155del4) in an African American with earlyonset breast cancer. Hum Mutat 14: 545, 1999.

13. Mefford HC, Baumbach L, Panguluri RC, et al: Evidence for a BRCA1 founder mutation in families of West African ancestry. Am J Hum Genet 65: 575-578, 1999.

14. Gao Q, Adebamowo CA, Fackenthal J, et al: Protein truncating BRCA1 and BRCA2 mutations in African women with premenopausal breast cancer. Hum Genet 107: 192-194, 2000.

15. Gao Q, Tomlinson G, Das S, et al: Prevalence of BRCA1 and BRCA2 mutations among clinic-based African American families with breast cancer. Hum Genet 107: 186-191, 2000.

16. Shen D, Wu Y, Subbarao M, Bhat H, Chillar R and Vadgama JV: Mutation analysis of BRCA1 gene in African-American patients with breast cancer. J Natl Med Assoc 92: 29-35, 2000.

17. Nanda R, Schumm LP, Cummings S, et al: Genetic testing in an ethnically diverse cohort of high-risk women: a comparative analysis of BRCA1 and BRCA2 mutations in American families of European and African ancestry. JAMA 294: 1925-1933, 2005.

18. Haffty DH. Choi S. Goyal, A, et al: Breast cancer in young women (YBC): prevalence of BRCA1/2 mutations and risk of secondary malignancies across diverse racial groups. Ann Oncol 20: 1653-1659, 2009.

19. Neuhausen SL, Godwin AK, Gershoni-Baruch R, et al: Haplotype and phenotype analysis of nine recurrent BRCA2 mutations in 111 families: results of an international study. Am J Hum Genet 62: 1381-1388, 1998.

20. El-Tamer MB and Wait RB: Age at presentation of AfricanAmerican and Caucasian breast cancer patients. J Am Coll Surg 188: 237-240, 1999.

21. Jemal A, Siegel R, Ward E, et al: Cancer statistics. CA Cancer J Clin 56: 106-130, 2006.

22. International Agency for Research on Cancer: GLOBOCAN 2002. http:// www-dep.iarc.fr/.

23. MacMahon B: Epidemiology and the causes of breast cancer. Int J Cancer 118: 2373-2378, 2006.

24. Adebamowo CA and Adekunle OO: Case-controlled study of the epidemiological risk factors for breast cancer in Nigeria. Br J Surg 86: 665-668, 1999

25. Eley JW, Hill HA, Chen VW, et al: Racial differences in survival from breast cancer: results of the National Cancer Institute Black/White Cancer Survival Study. JAMA 272: 947-954, 1994.

26. John EM, Miron A, Gong G, et al: Prevalence of pathogenic BRCA1 mutation carriers in 5 US racial/ethnic groups. JAMA 298: 2869-2876, 2007

27. Carey LA, Perou CM, Livasy CA, et al: Race, breast cancer subtypes, and survival in the Carolina Breast Cancer Study. JAMA 295: 2492- 2502, 2006.

28. Kurian AW, Gong GD, John EM, et al: Performance of prediction models for BRCA mutation carriage in three racial/ ethnic groups: findings from the Northern California Breast Cancer Family Registry. Cancer Epidemiol Biomarkers Prev 18: 1084-1091, 2009 